\title{
Global conservative and multipeakon conservative solutions for the two-component Camassa-Holm system
}

\author{
Yujuan Wang and Yongduan Song*
}

\section{"Correspondence:}

ydsong@cqu.edu.cn

School of Automation, Chongqing

University, Chongqing, 400044,

P.R. China

\begin{abstract}
The continuation of solutions for the two-component Camassa-Holm system after wave breaking is studied in this paper. The global conservative solution is derived first, from which a semigroup and a multipeakon conservative solution are established. In developing the solution, a system transformation based on a skillfully defined characteristic and a set of newly introduced variables is used. It is the transformation, together with the associated properties, that allows for the establishment of the results for continuity of the solution beyond collision time.
\end{abstract}

Keywords: two-component Camassa-Holm system; Lagrangian system; global conservative solutions; conservative multipeakon solutions

\section{Introduction}

Because of its capabilities of describing the dynamic behavior of water wave, the following Camassa-Holm $(\mathrm{CH})$ equation

$$
u_{t}-u_{x x t}+3 u u_{x}=2 u_{x} u_{x x}+u u_{x x x}
$$

modeling the unidirectional propagation of shallow water waves in irrotational flow over a flat bottom, with $u(t, x)$ representing the fluid velocity at time $t$ in the horizontal direction, has attracted considerable attention [1-10]. The $\mathrm{CH}$ equation is a quadratic order water wave equation in an asymptotic expansion for unidirectional shallow water waves described by the incompressible Euler equations, which was found earlier by Fuchssteiner and Fokas [1] as a bi-Hamiltonian generalization of the KdV equation. It is completely integrable $[2,3]$ and possesses an infinite number of conservation laws. A remarkable property of the $\mathrm{CH}$ equation is the existence of the non-smooth solitary wave solutions called peakons $[2,11]$. The peakon $u(t, x)=c e^{-|x-c t|}, c \neq 0$, is smooth except at its crest and the tallest among all waves of fixed energy. Another remarkable fact for the $\mathrm{CH}$ equation is that it can model wave breaking $[2,12]$, which means that the solution remains bounded while its slope becomes unbounded in finite time $[12,13]$, setting it apart from the classical soliton equations such as $\mathrm{KdV}$. After wave breaking, the solutions of the $\mathrm{CH}$ equation can be continued uniquely as either global conservative [4-6] or global dissipative solutions [7].

(0) 2013 Wang and Song; licensee Springer. This is an Open Access article distributed under the terms of the Creative Commons Attribution License (http://creativecommons.org/licenses/by/2.0), which permits unrestricted use, distribution, and reproduction in any medium, provided the original work is properly cited. 
Considered herein is the two-component Camassa-Holm $(\mathrm{CH} 2)$ shallow water system [14-16]

$$
\begin{cases}m_{t}+u m_{x}+2 u_{x} m+\sigma \rho \rho_{x}=0, & t>0, x \in R, \\ \rho_{t}+(u \rho)_{x}=0, & t>0, x \in R, \\ u(0, x)=u_{0}(x), & x \in R, \\ \rho(0, x)=\rho_{0}(x), & x \in R,\end{cases}
$$

with $\sigma=1$ and $m=\sigma_{1} u-u_{x x}, \sigma_{1}=1$ (or in the 'short wave' limit, $\sigma_{1}=0$ ), which is an extension of the $\mathrm{CH}$ equation by combining its integrability property with compressibility or free-surface elevation dynamics in its shallow water interpretation [11,17]. This system appeared originally in [14] as could be identified with the first negative flow of AKNS hierarchy, and then it was derived by Constantin and Ivanov [16] in the context of shallow water theory, with $u(x, t)$ representing the horizontal velocity of the fluid and $\rho(x, t)$ in connection with the free-surface elevation from equilibrium with the boundary assumptions $u \rightarrow 0$ and $\rho \rightarrow 1$ as $|x| \rightarrow \infty$. It is formally integrable [14-16] in the sense that it can be written as a compatibility condition of two linear systems (Lax pair) with a spectral parameter $\zeta$ :

$$
\Psi_{x x}=\left(-\sigma \zeta^{2} \rho^{2}+\zeta m+\frac{\sigma_{1}}{4}\right) \Psi, \quad \Psi_{t}=\left(\frac{1}{2 \zeta}-u\right) \Psi_{x}+\frac{1}{2} u_{x} \Psi
$$

It also has a bi-Hamiltonian structure corresponding to the Hamiltonian

$$
H_{1}=\frac{1}{2} \int\left(u m+(\rho-1)^{2}\right) d x
$$

and the Hamiltonian

$$
H_{2}=\frac{1}{2} \int\left(u(\rho-1)^{2}+2 u(\rho-1)+u^{3}+u u_{x}^{2}\right) d x .
$$

The Cauchy problem for the two-component Camassa-Holm system has been studied extensively [18-24]. It was shown that the $\mathrm{CH} 2$ system is locally well posed with initial data $\left(u_{0}, \rho_{0}\right) \in H^{s} \times H^{s-1}, s>\frac{3}{2}$ [18]. The system also has global strong solutions which blow-up in finite time [19, 21, 22] and a global weak solution [23]. However, the problem about continuation of the solutions beyond wave breaking, although interesting and important, has not been explicitly addressed yet. In our recent work [20], we studied the continuation beyond wave breaking by applying an approach that reformulated system (1.2) as a semilinear system of O.D.E. taking values in a Banach space. Such treatment makes it possible to investigate the continuity of the solution beyond collision time, leading to a global conservative solution where the energy is conserved for almost all times.

It should be stressed that both global conservation and multipeakon conservation are two important aspects worthy of investigation. To our best knowledge, however, little effort has been made in studying the multipeakon conservation associated with the $\mathrm{CH} 2$ system in the literature. As a compliment and extension to the previous work [20], we develop a novel approach in this work to construct the multipeakon conservative solution for the $\mathrm{CH} 2$ system. Different from the work [20], we reformulate the problem by utilizing 
a skillfully defined characteristic and a new set of variables, of which the associated energy serves as an additional variable to be introduced such that a well-posed initial-value problem can be obtained, making it convenient to study the dynamic behavior of wave breaking. Because of the introduction of the new variables, we are able to establish the multipeakon conservative solution from the global conservative solution for the $\mathrm{CH} 2$ system.

Some related earlier works $[4,5]$ studied the global existence of solutions to the $\mathrm{CH}$ equation. However, the system considered in this work is a heavily coupled one, in which the mutual effect between the two components makes the analysis quite complicated and involved as compared with the system with a single component as studied in $[4,5]$. The key and novel effort made in this work to circumvent the difficulty is the utilization of the skillfully defined characteristic and the new set of variables, as well as careful estimates for each iterative approximate component of the solutions, which allows us to establish the global conservative solutions of system (1.2). It is shown that the multipeakon structure is preserved by the semigroup of a global conservative solution and the multipeakon solution is obtained by carefully computing the convolution equations $P_{i}$ and $P_{x i}(i=1, \ldots, n)$, where, in contrast to the existing works, the inherent mutual effect between the two components is well reflected.

The remainder of this paper is organized as follows. Section 2 presents the transformation from the original system to a Lagrangian semilinear system. The global solutions of the equivalent semilinear system are obtained in Section 3, which are transformed into the global conservative solutions of the original system in Section 4. Finally, we establish the multipeakon conservative solutions for the original system in Section 5.

\section{The original system and the equivalent Lagrangian system}

We first present the original system. For simplicity, we consider here the associated evolution for positive times (of course, one would get similar results for negative times just by changing the initial condition $u_{0}$ into $\left.-u_{0}\right)$. Let us introduce an operator $\Lambda=\left(1-\partial_{x}^{2}\right)^{-1}$, which can be expressed by its associated Green's function $G=\frac{1}{2} e^{-|x|}$ such as $\Lambda f(x)=$ $G * f(x)=\frac{1}{2} \int_{R} e^{-\left|x-x^{\prime}\right|} f\left(x^{\prime}\right) d x^{\prime}$ for all $f \in L^{2}(R)$. Thus, we can rewrite Eq. (1.2) as a form of a quasi-linear evolution equation:

$$
\begin{cases}u_{t}+u u_{x}+\partial_{x} G *\left(u^{2}+\frac{1}{2} u_{x}^{2}+\frac{1}{2} v^{2}+v\right)=0, & t>0, x \in R, \\ v_{t}+(u v)_{x}+u_{x}=0, & t>0, x \in R, \\ u(0, x)=u_{0}(x), & x \in R, \\ v(0, x)=v_{0}(x), & x \in R,\end{cases}
$$

where $v=\rho-1$. If we define $P$ as

$$
P(t, x)=G *\left(u^{2}+\frac{1}{2} u_{x}^{2}+\frac{1}{2} v^{2}+v\right)=\frac{1}{2} \int_{R} e^{-\left|x-x^{\prime}\right|}\left(u^{2}+\frac{1}{2} u_{x}^{2}+\frac{1}{2} v^{2}+v\right)\left(t, x^{\prime}\right) d x^{\prime},
$$

then Eq. (1.2) can be rewritten as

$$
\begin{cases}u_{t}+u u_{x}+P_{x}=0, & t>0, x \in R, \\ v_{t}+u v_{x}+v u_{x}+u_{x}=0, & t>0, x \in R, \\ u(0, x)=u_{0}(x), & x \in R, \\ v(0, x)=v_{0}(x), & x \in R .\end{cases}
$$


Moreover, for regular solutions, we have that the total energy

$$
E(t)=\int_{R} u^{2}+u_{x}^{2}+v^{2} d x
$$

is constant in time. Thus, Eq. (2.1) possesses the $H^{1}$-norm conservation law defined as

$$
\|z\|_{H^{1}}=\|u\|_{H^{1}}+\|v\|_{L^{2}}=\left(\int_{R}\left[u^{2}+u_{x}^{2}+v^{2}\right] d x\right)^{1 / 2}
$$

where $z=(u, v)$. Since $z=(u, v) \in H^{1} \times\left[L^{2} \cap L^{\infty}\right]$, Young's inequality ensures $P \in H^{1}$.

We reformulate system (2.1) into a Lagrangian equivalent semilinear system as follows.

Let $z(t, x)=(u, v)(t, x)$ denote the solution of system (2.1). For given initial data $y(0, \xi)$, we define the corresponding characteristic $y(t, \xi)$ as the solution of

$$
y_{t}(t, \xi)=u(t, y(t, \xi))
$$

and define the Lagrangian cumulative energy distribution $H$ as

$$
H(t, \xi)=\int_{-\infty}^{y(t, \xi)}\left(u^{2}+u_{x}^{2}+v^{2}\right)(t, x) d x
$$

It is not hard to check that

$$
\left(u^{2}+u_{x}^{2}+v^{2}\right)_{t}+\left(u\left(u^{2}+u_{x}^{2}+v^{2}\right)\right)_{x}=\left(u^{3}-2 u P\right)_{x} .
$$

Then it follows from (2.3) and (2.5) that

$$
\frac{d H}{d t}=\left[\left(u^{3}-2 u P\right)(t, y(t, \xi))\right]_{-\infty}^{\xi}
$$

Throughout the following, we use the notation

$$
U(t, \xi)=u(t, y(t, \xi)), \quad V(t, \xi)=v(t, y(t, \xi)), \quad N(t, \xi)=u_{x}(t, y(t, \xi))
$$

After the change of variables $x=y(t, \xi)$ and $x^{\prime}=y\left(t, \xi^{\prime}\right)$, we obtain the following expressions for $P_{x}$ and $P$, namely

$$
\begin{aligned}
& P(\xi)=P(y(\xi))=\frac{1}{2} \int_{R} e^{-\left|y(\xi)-y\left(\xi^{\prime}\right)\right|}\left[\left(U^{2}+\frac{1}{2} U_{x}^{2}+\frac{1}{2} V^{2}+V\right) y_{\xi}\right]\left(\xi^{\prime}\right) d \xi^{\prime} \\
& P_{x}(\xi)=P_{x}(y(\xi))=-\frac{1}{2} \int_{R} \operatorname{sgn}\left(\xi-\xi^{\prime}\right) e^{-\left|y(\xi)-y\left(\xi^{\prime}\right)\right|} \\
& {\left[\left(U^{2}+\frac{1}{2} U_{x}^{2}+\frac{1}{2} V^{2}+V\right) y_{\xi}\right]\left(\xi^{\prime}\right) d \xi^{\prime}}
\end{aligned}
$$

where we have dropped the variable $t$ for simplicity and taken that $y$ is an increasing function for any fixed time $t$ for granted (the validity will be proved later). Using 


$$
\begin{aligned}
H_{\xi}= & \left(u^{2}+u_{x}^{2}+v^{2}\right) \circ y y_{\xi}, \text { we can rewrite } P_{x} \text { and } P \text { in }(2.7) \text { as } \\
P_{x}(\xi) & =-\frac{1}{4} \int_{R} \operatorname{sgn}\left(\xi-\xi^{\prime}\right) e^{-\left|y(\xi)-y\left(\xi^{\prime}\right)\right|}\left[H_{\xi}+\left(U^{2}+2 V\right) y_{\xi}\right]\left(\xi^{\prime}\right) d \xi^{\prime} \\
& P(\xi)=\frac{1}{4} \int_{R} e^{-\left|y(\xi)-y\left(\xi^{\prime}\right)\right|}\left[H_{\xi}+\left(U^{2}+2 V\right) y_{\xi}\right]\left(\xi^{\prime}\right) d \xi^{\prime}
\end{aligned}
$$

From the definition of the characteristic, it follows that

$$
\begin{aligned}
& U_{t}(t, \xi)=u_{t}(t, y)+u_{x}(t, y) y_{t}(t, \xi)=-P_{x} \circ y(t, \xi), \\
& V_{t}(t, \xi)=v_{t}(t, y)+v_{x}(t, y) y_{t}(t, \xi)=-\left[(v+1) u_{x}\right] \circ y(t, \xi), \\
& N_{t}(t, \xi)=u_{x t}(t, y)+u_{x x}(t, y) y_{t}(t, \xi)=\left(u^{2}-\frac{1}{2} u_{x}^{2}+\frac{1}{2} v^{2}+v-P\right) \circ y(t, \xi) .
\end{aligned}
$$

Let us introduce another variable $\varsigma(t, \xi)$ such that $\varsigma(t, \xi)=y(t, \xi)-\xi$ (it will turn out that $\varsigma \in L^{\infty}(R)$ ). With these new variables, we now derive an equivalent system of equations (2.1),

$$
\left\{\begin{array}{l}
\varsigma_{t}=U \\
U_{t}=-P_{x} \\
V_{t}=-(V+1) N \\
N_{t}=U^{2}-\frac{1}{2} N^{2}+\frac{1}{2} V^{2}+V-P \\
H_{t}=U^{3}-2 U P
\end{array}\right.
$$

where $P$ and $P_{x}$ are given by (2.8). Differentiating (2.10) w.r.t. $\xi$ yields

$$
\left\{\begin{array}{l}
\varsigma_{\xi t}=U_{\xi}, \\
U_{\xi t}=\frac{1}{2} H_{\xi}+\left(\frac{1}{2} U^{2}+V-P\right) y_{\xi}, \\
V_{\xi t}=-V_{\xi} N-(V+1) N_{\xi}, \\
N_{\xi t}=2 U U_{\xi}+(V+1) V_{\xi}-N N_{\xi}-P_{x} y_{\xi}, \\
H_{\xi t}=\left(3 U^{2}-2 P\right) U_{\xi}-2 U P_{x} y_{\xi},
\end{array}\right.
$$

which is semilinear w.r.t. the variables $y_{\xi}, U_{\xi}, V_{\xi}, N_{\xi}$ and $H_{\xi}$.

System (2.10) can be regarded as an O.D.E. in the Banach space $E$ given by

$$
E=W \times H^{1} \times\left[L^{2} \cap L^{\infty}\right] \times\left[L^{2} \cap L^{\infty}\right] \times W,
$$

endowed with the norm

$$
\|X\|_{E}=\|\varsigma\|_{W}+\|U\|_{H^{1}}+\|V\|_{L^{2}}+\|V\|_{L^{\infty}}+\|N\|_{L^{2}}+\|N\|_{L^{\infty}}+\|H\|_{W}
$$

for any $X=(\varsigma, U, V, N, H) \in E$. Here $W$ is a Banach space defined as

$$
W=\left\{f \in C(R) \cap L^{\infty}(R) \mid f_{\xi} \in L^{2}(R)\right\},
$$

with the norm $\|f\|_{W}=\|f\|_{L^{\infty}(R)}+\left\|f_{\xi}\right\|_{L^{2}(R)}$. Note that $H^{1}(R) \subset W$. 


\section{Global solutions of the equivalent system}

In this section, we prove that the equivalent system admits a unique global solution. We first obtain the Lipschitz bounds we need on $P$ and $P_{x}$.

Lemma 3.1 (See [5]) Let $\Re_{1}: E \rightarrow W$ and $\Re_{2}: E \rightarrow H^{1}$, or $\Re_{2}: E \rightarrow W$ be two locally Lipschitz maps. Then the product $X \rightarrow \Re_{1}(X) \Re_{2}(X)$ is also a locally Lipschitz map from $E$ to $H^{1}$, or from $E$ to $W$.

Lemma 3.2 For any given $X=(\varsigma, U, V, N, H) \in E, P$ and $P_{x}$ defined by (2.8) are locally Lipschitz continuous from $E$ to $H^{1}(R)$. Moreover, we have

$$
P_{x \xi}=-\frac{1}{2} H_{\xi}+\left(P-\frac{1}{2} U^{2}-V\right)\left(1+\varsigma_{\xi}\right), \quad P_{\xi}=P_{x}\left(1+\varsigma_{\xi}\right) .
$$

Proof We write

$$
\begin{aligned}
P_{x}(\xi)= & P_{x}^{1}(X)(\xi)+P_{x}^{2}(X)(\xi) \\
= & -\frac{e^{-\zeta(\xi)}}{4} \int_{R} \chi_{\left\{\xi^{\prime}<\xi\right\}} e^{-\left|\xi-\xi^{\prime}\right|} e^{\zeta\left(\xi^{\prime}\right)}\left[H_{\xi}+\left(U^{2}+2 V\right)(1+\zeta \xi)\right]\left(\xi^{\prime}\right) d \xi^{\prime} \\
& +\frac{e^{\zeta(\xi)}}{4} \int_{R} \chi_{\left\{\xi^{\prime}>\xi\right\}} e^{-\left|\xi-\xi^{\prime}\right|} e^{-\zeta\left(\xi^{\prime}\right)}\left[H_{\xi}+\left(U^{2}+2 V\right)(1+\zeta \xi)\right]\left(\xi^{\prime}\right) d \xi^{\prime},
\end{aligned}
$$

where $\chi_{\Omega}$ denotes the indicator function of a given set $\Omega$, and $P_{x}^{1}, P_{x}^{2}$ are the operators which correspond to the two terms of the last identity in (3.2). We rewrite $P_{x}^{1}$ as

$$
P_{x}^{1}(X)(\xi)=-\frac{e^{-\zeta(\xi)}}{2} \Lambda \circ R(X)(\xi)
$$

where $R$ is the operator from $E$ to $L^{2}(R)$ given by

$$
R(X)(\xi)=\chi_{\left\{\xi^{\prime}<\xi\right\}} e^{\varsigma}\left[H_{\xi}+\left(U^{2}+2 V\right)(1+\varsigma \xi)\right] .
$$

Since the operator $\Lambda$ (defined as in Section 2) is linear and continuous from $H^{-1}(R)$ to $H^{1}(R)$, and $L^{2}(R)$ is continuously embedded in $H^{-1}(R)$, we have $\Lambda \circ R(X) \in H^{1}$. It is not hard to know that $R$ is locally Lipschitz from $E$ into $L^{2}(R)$ and therefore from $E$ into $H^{-1}(R)$. Thus, $\Lambda \circ R$ is locally Lipschitz from $E$ to $H^{1}(R)$. Since the mapping $X \rightarrow e^{-\varsigma}$ is locally Lipschitz from $E$ to $W$, it then follows from Lemma 3.1 that $P_{x}^{1}$ is locally Lipschitz from $E$ to $H^{1}(R)$. Similarly, $P_{x}^{2}$ is also locally Lipschitz and therefore $P_{x}$ is locally Lipschitz from $E$ to $H^{1}(R)$. We can obtain that $P$ defined by (2.8) is locally Lipschitz continuous from $E$ to $H^{1}(R)$ in the same way. By using the chain rule, the formulas in (3.1) are obtained by direct computation, see [25, p.129].

Theorem 3.1 Let any $\bar{X}=(\bar{S}, \bar{U}, \bar{V}, \bar{N}, \bar{H}) \in$ E be given. System (2.10) admits a unique local solution defined on some time interval $[0, T]$, where T depends only on $\|\bar{X}\|_{E}$.

Proof To establish the local existence of solutions, one proceeds as in Lemma 3.2, then obtains that $F(X)$, which is defined by

$$
F(X)=\left(U,-P_{x},-(V+1) N, U^{2}-\frac{1}{2} N^{2}+\frac{1}{2} V^{2}+V-P, U^{3}-2 U P\right)
$$


with $X=(\varsigma, U, V, N, H)$, is Lipschitz continuous on any bounded set of $E$. We rewrite the solutions of system (2.10) as

$$
X(t)=\bar{X}+\int_{0}^{t} F(X(\tau)) d \tau
$$

Then the theorem follows from the standard contraction argument on Banach spaces.

Theorem 3.1 gives us the existence of local solutions to (2.10) for initial data in $E$. It remains to prove that the local solutions can be extended to global solutions. Note that the global solutions of (2.10) may not exist for all initial data in $E$. However, they exist when the initial data $\bar{X}=(\bar{\zeta}, \bar{U}, \bar{V}, \bar{N}, \bar{H})$ belongs to the set $\Gamma$, which is defined as follows.

Definition 3.1 The set $\Gamma$ is composed of all $(\varsigma, U, V, N, H) \in E$ such that

(i) $(\varsigma, U, V, N, H) \in\left[W^{1, \infty}(R)\right]^{5}$,

(ii) $y_{\xi} \geq 0, H_{\xi} \geq 0, y_{\xi}+H_{\xi}>0$ almost everywhere, and $\lim _{\xi \rightarrow-\infty} H(\xi)=0$,

(iii) $y_{\xi} H_{\xi}=y_{\xi}^{2} U^{2}+U_{\xi}^{2}+y_{\xi}^{2} V^{2} \quad$ almost everywhere,

with $\varsigma(\xi)=y(\xi)-\xi$, where $W^{1, \infty}(R)=\left\{f \in C(R) \cap L^{\infty}(R) \mid f_{\xi} \in L^{\infty}(R)\right\}$.

The global existence of the solution for initial data in $\Gamma$ relies essentially on the fact that the set $\Gamma$ is preserved by the flow as the next lemma shows.

Lemma 3.3 Given initial data $\bar{X}=(\bar{\zeta}, \bar{U}, \bar{V}, \bar{N}, \bar{H}) \in \Gamma$, for some $T>0$, we consider the local solution $X(t)=(\varsigma, U, V, N, H)(t) \in C([0, T], E)$ of system (2.10) given by Theorem 3.1. We have

(i) $X(t) \in \Gamma$ for all $t \in[0, T]$,

(ii) $y_{\xi}(t, \xi)>0$ for a.e. $t \in[0, T]$ and a.e. $\xi \in R$,

(iii) $\lim _{\xi \rightarrow \pm \infty} H(t, \xi)=H(0, \pm \infty)=0$ for all $t \in[0, T]$.

Proof (i) For given initial data $\bar{X}=(\bar{\zeta}, \bar{U}, \bar{V}, \bar{N}, \bar{H}) \in E \cap\left[W^{1, \infty}(R)\right]^{5}$, to ensure that the solution $X=(\varsigma, U, V, N, H)$ of (2.10) also belongs to $E \cap\left[W^{1, \infty}(R)\right]^{5}$, we have to specify the initial conditions for (2.11). Let $\Omega$ be the following set:

$$
\begin{aligned}
\Omega= & \left\{\xi \in R|| \bar{\zeta}_{\xi}(\xi)\left|\leq\left\|\bar{\zeta}_{\xi}\right\|_{L^{\infty}},\right| \bar{U}_{\xi}(\xi)\left|\leq\left\|\bar{U}_{\xi}\right\|_{L^{\infty}},\right| \bar{V}_{\xi}(\xi) \mid \leq\left\|\bar{V}_{\xi}\right\|_{L^{\infty}},\right. \\
& \left.\left|\bar{N}_{\xi}(\xi)\right| \leq\left\|\bar{N}_{\xi}\right\|_{L^{\infty}},\left|\bar{H}_{\xi}(\xi)\right| \leq\left\|\bar{H}_{\xi}\right\|_{L^{\infty}}\right\} .
\end{aligned}
$$

Note that meas $\left(\Omega^{c}\right)=0$. For $\xi \in \Omega$, we take $\left(\varsigma_{\xi}, U_{\xi}, V_{\xi}, N_{\xi}, H_{\xi}\right)(0, \xi)=\left(\bar{\zeta}_{\xi}, \bar{U}_{\xi}, \bar{V}_{\xi}, \bar{N}_{\xi}\right.$, $\left.\bar{H}_{\xi}\right)(\xi)$. For $\xi \in \Omega^{c}$, we define $\left(\varsigma_{\xi}, U_{\xi}, V_{\xi}, N_{\xi}, H_{\xi}\right)(0, \xi)=(0,0,0,0,0)$. We consider $U, P$ and $P_{x}$ as given functions in $C\left([0, T], H^{1}(R)\right)$, which is guaranteed by Lemma 3.2 and $V$, $N$ in $C\left([0, T],\left[L^{2}(R) \cap L^{\infty}(R)\right]\right)$. Thus system (2.11) is affine (it consists of a sum of a linear transformation and a constant) and, therefore, by using a contraction argument, it admits a unique local solution defined on some time interval $[0, T]$. Thus, for the given initial condition $\bar{X} \in E \cap\left[W^{1, \infty}(R)\right]^{5}$, the solution of (2.10) given by Theorem 3.1 also belongs to $E \cap\left[W^{1, \infty}(R)\right]^{5}$, which implies that $X(t)$ satisfies (3.5a) for all $t \in[0, T]$. We claim that 
(3.5c) holds for any $\xi \in \Omega$ and therefore almost everywhere. Consider a fixed $\xi \in \Omega$ and drop it in the notation. On the one hand, it follows from (2.11) that

$$
\begin{aligned}
\left(y_{\xi} H_{\xi}\right)_{t} & =y_{\xi t} H_{\xi}+y_{\xi} H_{\xi t} \\
& =U_{\xi} H_{\xi}+y_{\xi}\left[\left(3 U^{2}-2 P-2 A U\right) U_{\xi}-2 U P_{x} y_{\xi}\right] \\
& =U_{\xi} H_{\xi}+3 U^{2} U_{\xi} y_{\xi}-2 P U_{\xi} y_{\xi}-2 A U U_{\xi} y_{\xi}-2 U P_{x} y_{\xi}^{2},
\end{aligned}
$$

and on the other hand,

$$
\begin{aligned}
& \left(y_{\xi}^{2} U^{2}+U_{\xi}^{2}+y_{\xi}^{2} V^{2}\right)_{t} \\
& \quad=2 y_{\xi} y_{\xi t} U^{2}+2 y_{\xi}^{2} U U_{t}+2 y_{\xi} y_{\xi t} V^{2}+2 y_{\xi}^{2} V V_{t}+2 U_{\xi} U_{\xi t} \\
& =3 U^{2} U_{\xi} y_{\xi}-2 U P_{x} y_{\xi}^{2}+U_{\xi} H_{\xi}-2 A U U_{\xi} y_{\xi}-2 P U_{\xi} y_{\xi} .
\end{aligned}
$$

Thus, $\left(y_{\xi} H_{\xi}\right)_{t}=\left(y_{\xi}^{2} U^{2}+U_{\xi}^{2}+y_{\xi}^{2} V^{2}\right)_{t}$. Notice that $y_{\xi} H_{\xi}(0)=\left(y_{\xi}^{2} U^{2}+U_{\xi}^{2}+y_{\xi}^{2} V^{2}\right)(0)$, which implies that $y_{\xi} H_{\xi}(t)=\left(y_{\xi}^{2} U^{2}+U_{\xi}^{2}+y_{\xi}^{2} V^{2}\right)(t)$ for all $t \in[0, T]$. Thus, (3.5c) holds. It remains to prove that the inequalities in (3.5b) hold. Set $t^{*}=\sup \left\{t \in[0, T] \mid y_{\xi}\left(t^{\prime}\right) \geq 0\right.$ for all $t^{\prime} \in$ $[0, t]\}$. Assume that $t^{*}<T$. Since $y_{\xi}(t)$ is continuous w.r.t. $t$, we have $y_{\xi}\left(t^{*}\right)=0$. It follows from (3.5c) that $U_{\xi}\left(t^{*}\right)=0$. Furthermore, (2.11) implies $y_{\xi t}\left(t^{*}\right)=U_{\xi}\left(t^{*}\right)=0$ and $y_{\xi t t}\left(t^{*}\right)=U_{\xi t}\left(t^{*}\right)=\frac{1}{2} H_{\xi}\left(t^{*}\right)$. If $H_{\xi}\left(t^{*}\right)=0$, then $\left(y_{\xi}, U_{\xi}, H_{\xi}\right)\left(t^{*}\right)=(0,0,0)$, which implies $\left(y_{\xi}, U_{\xi}, H_{\xi}\right)(t)=0$ for all $t \in[0, T]$ by the uniqueness of the solution of system (2.11). This contradicts the fact that $y_{\xi}(0)+H_{\xi}(0)>0$ for all $\xi \in \Omega$. If $H_{\xi}\left(t^{*}\right)<0$, then $y_{\xi t t}\left(t^{*}\right)<0$. Since $y_{\xi}\left(t^{*}\right)=y_{\xi t}\left(t^{*}\right)=0$, there exists a neighborhood $\varpi$ of $t^{*}$ such that $y_{\xi}(t)<0$ for all $t \in \varpi /\left\{t^{*}\right\}$. This contradicts the definition of $t^{*}$. Hence, $H_{\xi}\left(t^{*}\right)>0$. We now have $y_{\xi t t}\left(t^{*}\right)>0$, which conversely implies $y_{\xi}(t)>0$ for all $t \in \varpi /\left\{t^{*}\right\}$, which contradicts the fact that $t^{*}<T$. Thus, we have proved $y_{\xi}(t) \geq 0$ for all $t \in[0, T]$. We now prove that $H_{\xi} \geq 0$ for all $t \in[0, T]$. This follows from (3.5c) when $y_{\xi}(t)>0$. If $y_{\xi}(t)=0$, then $U_{\xi}(t)=0$ from (3.5c). As we have seen, $H_{\xi}<0$ would imply that $y_{\xi}\left(t^{\prime}\right)<0$ for some $t^{\prime}$ in a punctured neighborhood of $t$, which is impossible. Hence, $H_{\xi} \geq 0$ for all $t \in[0, T]$. Now we have $y_{\xi}(t)+H_{\xi}(t) \geq 0$ for all $t \in[0, T]$. If $y_{\xi}\left(t^{\prime}\right)+H_{\xi}\left(t^{\prime}\right)=0$ for some $t^{\prime}$, it then follows that $\left(y_{\xi}, U_{\xi}, H_{\xi}\right)\left(t^{\prime}\right)=0$, which implies $\left(y_{\xi}, U_{\xi}, H_{\xi}\right)(t)=0$ for all $t \in[0, T]$, which contradicts the fact that $y_{\xi}(0)+H_{\xi}(0)>0$ for all $\xi \in \Omega$. Hence, $y_{\xi}(t)+H_{\xi}(t)>0$.

(ii) Define the set $N=\left\{(t, \xi) \in[0, T] \times R \mid y_{\xi}(t, \xi)=0\right\}$. It follows from Fubini's theorem that

$$
\operatorname{meas}(N)=\int_{R} \operatorname{meas}\left(N_{\xi}\right) d \xi=\int_{[0, T]} \operatorname{meas}\left(N_{t}\right) d t,
$$

where $N_{\xi}=\left\{t \in[0, T] \mid y_{\xi}(t, \xi)=0\right\}$ and $N_{t}=\left\{\xi \in R \mid y_{\xi}(t, \xi)=0\right\}$. From the above proof, we know that for all $\xi \in \Omega, N_{\xi}$ consists of isolated points that are countable. This means that meas $\left(N_{\xi}\right)=0$. It follows from (3.6), and since meas $\left(\Omega^{c}\right)=0$, that

$$
\text { meas }\left(N_{t}\right)=0 \text { for almost every } t \in[0, T] .
$$

This implies that $y_{\xi}(t, \xi)>0$ for almost all $t$ and therefore $y(t, \xi)$ is strictly increasing and invertible w.r.t. $\xi$. 
(iii) For any given $t \in[0, T]$, since $H_{\xi}(t) \geq 0$ and $H(t, \xi) \in L^{\infty}(R)$, we know that $H(t, \pm \infty)$ exist. We have

$$
H(t, \xi)=H(0, \xi)+\int_{0}^{t}\left(U^{3}-2 P U\right)(\tau, \xi) d \tau
$$

Let $\xi \rightarrow \pm \infty$. Since $U, P$ are bounded in $L^{\infty}([0, T] \times R)$ and $\lim _{\xi \rightarrow \pm \infty} U(t, \xi)=0$ as $U(t, \cdot) \in H^{1}(R)$, it then follows from (3.7) that $H(t, \pm \infty)=H(0, \pm \infty)$ for all $t \in[0, T]$. Since $\bar{X} \in \Gamma$, it follows that $H(0, \pm \infty)=0$ for all $t \in[0, T]$.

Theorem 3.2 For any initial data $\bar{X}=(\bar{y}, \bar{U}, \bar{V}, \bar{N}, \bar{H}) \in \Gamma$, there exists a unique global solution $X(t)=(y, U, V, N, H)(t) \in C^{1}\left(R^{+}, E\right)$ for system (2.10). Moreover, for all $t \geq 0$, if we equip $\Gamma$ with the topology endowed with the E-norm, then the map $S_{t}: \Gamma \rightarrow \Gamma \times R^{+}$defined as

$$
S_{t}(\bar{X})=X(t)
$$

is a continuous semigroup.

Proof Let $(\varsigma, U, V, N, H) \in C([0, T], E)$ be a local solution of (2.10) with initial data $(\bar{\zeta}, \bar{U}, \bar{V}, \bar{N}, \bar{H})$. To obtain the global existence of solutions, it suffices to show that

$$
\sup _{t \in[0, T)}\|\varsigma(t, \cdot), U(t, \cdot), V(t, \cdot), N(t, \cdot), H(t, \cdot)\|_{E}<\infty
$$

Since $H(t, \xi)$ is an increasing function w.r.t. $\xi$ for all $t$ and $\lim _{\xi \rightarrow \infty} H(t, \xi)=\lim _{\xi \rightarrow \infty} H(0, \xi)$, we have $\sup _{t \in[0, T)}\|H(t, \cdot)\|_{L^{\infty}(R)}=\|\bar{H}\|_{L^{\infty}(R)}<\infty$. We consider a fixed $t \in[0, T)$ and drop it for simplification. Since $U_{\xi}(\xi)=0$ when $y_{\xi}(\xi)=0$, and $y_{\xi}(\xi)>0$ for a.e. $\xi$, it follows from (3.5c) that

$$
\begin{aligned}
U^{2}(\xi) & =2 \int_{-\infty}^{\xi} U\left(\xi^{\prime}\right) U_{\xi}\left(\xi^{\prime}\right) d \xi^{\prime}=2 \int_{\left\{\xi^{\prime}<\xi \mid y_{\xi}\left(\xi^{\prime}\right)>0\right\}} U\left(\xi^{\prime}\right) U_{\xi}\left(\xi^{\prime}\right) d \xi^{\prime} \\
& \leq \int_{\left\{\xi^{\prime}<\xi \mid y_{\xi}\left(\xi^{\prime}\right)>0\right\}}\left(y_{\xi} U^{2}+U_{\xi}^{2} / y_{\xi}\right)\left(\xi^{\prime}\right) d \xi^{\prime} \leq \int_{R} H_{\xi}\left(\xi^{\prime}\right) d \xi^{\prime}=H(\xi),
\end{aligned}
$$

which implies

$$
\sup _{t \in[0, T)}\left\|U^{2}(t, \cdot)\right\|_{L^{\infty}} \leq \sup _{t \in[0, T)}\|H(t, \cdot)\|_{L^{\infty}(R)}=\|\bar{H}\|_{L^{\infty}(R)}<\infty
$$

and therefore

$$
\sup _{t \in[0, T)}\|U(t, \cdot)\|_{L^{\infty}}<\infty
$$

We can obtain from the governing equation (2.10) that

$$
|\varsigma(t, \xi)| \leq|\varsigma(0, \xi)|+\sup _{t \in[0, T)}\|U(t, \cdot)\|_{L^{\infty}} T .
$$


Thus, $\sup _{t \in[0, T)}\|\varsigma(t, \cdot)\|_{L^{\infty}}<\infty$. The governing equation (2.10) also implies that $\sup _{t \in[0, T)}\|V(t, \cdot)\|_{L^{\infty}}<\infty$ and $\sup _{t \in[0, T)}\|N(t, \cdot)\|_{L^{\infty}}<\infty$.

From the identity $H_{\xi}=\left(U^{2}+U_{x}^{2}+V^{2}\right) y_{\xi}$, we can deduce that

$$
\left|\left(U^{2}+2 V\right) y_{\xi}\right| \leq\left(U^{2}+V^{2}+1\right) y_{\xi} \leq H_{\xi}+y_{\xi}
$$

which implies that

$$
\begin{aligned}
\left|P_{x}\right| & \leq \frac{1}{2}\left|\int_{R} e^{-\left|y(\xi)-y\left(\xi^{\prime}\right)\right|}\left[H_{\xi}+\left(U^{2}+2 V\right) y_{\xi}\right]\left(\xi^{\prime}\right) d \xi^{\prime}\right| \\
& \leq \frac{1}{2}\left|\int_{R} e^{-\left|y(\xi)-y\left(\xi^{\prime}\right)\right|}\left[2 H_{\xi}+y_{\xi}\right]\left(\xi^{\prime}\right) d \xi^{\prime}\right| \\
& \leq C\left(\sup _{t \in[0, T)}\|H(t, \cdot)\|_{L^{\infty}(R)}+\sup _{t \in[0, T)}\|\varsigma(t, \cdot)\|_{L^{\infty}}\right)<\infty .
\end{aligned}
$$

Therefore, $\left\|P_{x}\right\|_{L^{\infty}}<\infty$. Similarly, we obtain $\left\|P_{x}\right\|_{L^{2}}<\infty$ and the bounds hold for $P$. Let

$$
Z(t)=\|U(t, \cdot)\|_{L^{2}}+\left\|U_{\xi}(t, \cdot)\right\|_{L^{2}}+\|V(t, \cdot)\|_{L^{2}}+\|N(t, \cdot)\|_{L^{2}}+\left\|\zeta_{\xi}(t, \cdot)\right\|_{L^{2}}+\left\|H_{\xi}(t, \cdot)\right\|_{L^{2}} \cdot
$$

After taking the $L^{2}$-norms on both sides of (2.10) and (2.11), we obtain

$$
Z(t) \leq Z(0)+C \int_{0}^{t} Z(\tau) d \tau
$$

It follows from Gronwall's lemma that $\sup _{t \in[0, T)} Z(t)<\infty$, which implies that $S_{t}$ is a continuous semigroup by the standard O.D.E. theory.

\section{Global solutions for the original system}

We transform the global solution of the equivalent system (2.10) into the global conservative solution of the original system (2.1) in this section. It suffices to establish the correspondence between the Lagrangian equivalent system and the original system.

We first introduce a set $G$ as the set of relabeling functions defined by

$$
G=\left\{f \text { is invertible } f-\operatorname{Id} \text { and } f^{-1}-\operatorname{Id} \text { both belong to } W^{1, \infty}(R)\right\},
$$

where Id denotes the identity function. For any $\alpha>1$, we define the subsets $G_{\alpha}$ of $G$ as

$$
G_{\alpha}=\left\{f \in G \mid\|f-\mathrm{Id}\|_{W^{1, \infty}(R)}+\left\|f^{-1}-\mathrm{Id}\right\|_{W^{1, \infty}(R)} \leq \alpha\right\},
$$

with a useful property: If $f \in G_{\alpha}(\alpha \geq 0)$, then $1 /(1+\alpha) \leq f_{\xi} \leq 1+\alpha$ almost everywhere. Conversely, if $f$ is absolutely continuous, $f-\operatorname{Id} \in L^{\infty}(R)$ and there exists $c \geq 1$ such that $1 / c \leq f_{\xi} \leq c$ almost everywhere, then $f \in G_{\alpha}$ for some $\alpha$ depending only on $c$ and $\| f-$ Id $\|_{L^{\infty}(R)}$. We now define the subsets $F$ and $F_{\alpha}$ of $\Gamma$ such that

$$
\begin{aligned}
& F=\{X=(y, U, V, H) \in \Gamma \mid y+H \in G\}, \\
& F_{\alpha}=\left\{X=(y, U, V, H) \in \Gamma \mid y+H \in G_{\alpha}\right\} .
\end{aligned}
$$


With the above useful property of $G_{\alpha}$, it is not hard to prove that the space $F$ is preserved by the governing equation (2.10).

Notice that the map $\Phi: G \times F \rightarrow F$ given by $\Phi(f, X)=X \circ f$ defines a group action of $G$ on $F$, we then consider the quotient space $F / G$ of $F$ w.r.t. the group action. The equivalence relation on $F$ is defined as: for any $X, X^{\prime} \in F$, if there exists $f \in G$ such that $X^{\prime}=X \circ f$, we claim that $X$ and $X^{\prime}$ are equivalent. We denote the projection $\Pi: F \rightarrow F / G$ by $\Pi(X)=[X]$. For any $X=(y, U, V, N, H) \in F$, we introduce the map $K: F \rightarrow F_{0}$ given by $K(X)=X$ 。 $(y+H)^{-1}$. It is not hard to prove that $K(X)=X$ when $X \in F_{0}$, and $K(X \circ f)=K(X)$ for any $X \in F$ and $f \in G$. Hence, we can define the map $\tilde{K}: F / G \rightarrow F_{0}$ as $\tilde{K}([X])=K(X)$ for any representative $[X] \in F / G$ of $X \in F$. For any $X \in F_{0}$, we have $\tilde{K} \circ \Pi(X)=K(X)=X$. Hence, $\tilde{K} \circ \Pi_{\mid F_{0}}=\operatorname{Id}_{\mid F_{0}}$. Note that any topology defined on $F_{0}$ is naturally transported into $F / G$ by this isomorphism, that is, if we equip $F_{0}$ with the metric induced by the $E$-norm, i.e., $d_{F_{0}}\left(X, X^{\prime}\right)=\left\|X-X^{\prime}\right\|_{E}$ for all $X, X^{\prime} \in F_{0}$, which is complete, then for any $[X],\left[X^{\prime}\right] \in F / G$, the topology on $F / G$ is defined by a complete metric given by $d_{F / G}\left([X],\left[X^{\prime}\right]\right)=\| K(X)-$ $K\left(X^{\prime}\right) \|_{E}$.

For any initial data $\bar{X} \in F$, we denote the continuous semigroup with the solution $X(t)$ of system (2.10) by $S: F \times R^{+} \rightarrow F$. As we indicated earlier, Eq. (2.1) is invariant w.r.t. relabeling. That is, $t>0, S_{t}(X \circ f)=S_{t}(X) \circ f$ for any $X \in F$ and $f \in G$. Thus, the map $\tilde{S}_{t}$ : $F / G \rightarrow F / G$ defined by $\tilde{S}_{t}([X])=\left[S_{t} X\right]$ is valid, which generates a continuous semigroup.

To derive the correspondence between the Lagrangian equivalent system and the original system, we have to consider the space $D$, which characterizes the solutions in the original system:

$$
D=\left\{(z, \mu) \mid z \in H^{1}(R) \times\left[L^{2}(R) \times L^{\infty}(R)\right] \text { and } \mu_{\mathrm{ac}}=\left(u^{2}+u_{x}^{2}+v^{2}\right) d x\right\},
$$

where $z=(u, v)$ and $\mu$ is a positive finite Radon measure with $\mu_{\mathrm{ac}}$ as its absolute continuous part.

We now establish a bijection between $F / G$ and $D$ to transport the continuous semigroup obtained in the Lagrangian equivalent system (functions in $F / G$ ) into the original system (functions in $D$ ).

We first introduce the mapping $M$, which corresponds to the transformation from the Lagrangian equivalent system into the original system. In the other direction, we obtain the energy density $\mu$ in the original system, by pushing forward by $y$ the energy density $H_{\xi} d \xi$ in the Lagrangian equivalent system, where the push-forward $f_{\#} v$ of a measure $v$ by a measurable function $f$ is defined by

$$
f_{\#} v(B)=v\left(f^{-1}(B)\right)
$$

for all Borel set $B$. Let $(z, \mu)$ be defined as

$$
\begin{aligned}
& z(x)=Z(\xi) \quad \text { for any } \xi \text { such that } x=y(\xi), \\
& \mu=y_{\#}\left(H_{\xi} d \xi\right)
\end{aligned}
$$

where $z(x)=(u, v)(x), Z(\xi)=(U, V)(\xi)$. We have that $(z, \mu) \in D$, which does not depend on the representative $X=(y, U, V, N, H) \in F$ of $[X]$ we choose. We denote by $M: F / G \rightarrow D$ 
the mapping to any $[X] \in F / G$ and $(z, \mu) \in D$ given by (4.1a) and (4.1b), which transforms the Lagrangian equivalent system into the original system.

We are led to the mapping $L: D \rightarrow F / G$, which conversely transforms the original system into the Lagrangian equivalent system defined as follows.

Definition 4.1 For any $(z, \mu) \in D$, let

$$
\begin{aligned}
& y(\xi)=\sup \{y \mid \mu(-\infty, y)+y<\xi\}, \\
& U(\xi)=u \circ y(\xi), \quad V(\xi)=v \circ y(\xi), \quad N(\xi)=u_{x} \circ y(\xi), \\
& H(\xi)=\xi-y(\xi),
\end{aligned}
$$

where $z=(u, v)$. We define $L(z, \mu) \in F / G$ as the equivalence class of $(y, U, V, N, H)$.

Remark 4.1 Note that $X=(y, U, V, N, H) \in E$, which satisfies (3.5a)-(3.5c) from the definition of $y, U, V, N, H$ in (4.2a)-(4.2c). Moreover, by the definition (4.2c), we have that $y+H=$ Id. Thus, $X=(y, U, V, N, H) \in F_{0}$.

We claim that the transformation from the original system into the Lagrangian equivalent system is a bijection.

Theorem 4.1 The maps $M$ and $L$ are invertible, that is,

$$
L \circ M=\operatorname{Id}_{F / G}, \quad M \circ L=\operatorname{Id}_{D}
$$

Proof Let $[X] \in F / G$ be given. We consider $X=(y, U, V, N, H)=\tilde{K}([X])$ for a representative of $[X]$ and $(z, \mu)$ given by (4.1a) and (4.1b) for this particular $X$. From the definition of $\tilde{K}$, we have $X \in F_{0}$. Let $\bar{X}=(\bar{y}, \bar{U}, \bar{V}, \bar{N}, \bar{H})$ be the representative of $L(z, \mu)$ in $F_{0}$ given by (4.2a)-(4.2c). To derive $L \circ M=\operatorname{Id}_{F / G}$, it suffices to show that $(\bar{y}, \bar{U}, \bar{V}, \bar{N}, \bar{H})=$ $(y, U, V, N, H)$. Let

$$
g(x)=\sup \{\xi \in R \mid y(\xi)<x\} .
$$

Using the fact that $y$ is increasing and continuous, it follows that

$$
y(g(x))=x
$$

and $y^{-1}((-\infty, x))=(-\infty, g(x))$. From (4.1b) and since $H(-\infty)=0$, for any $x \in R$, we get

$$
\mu((-\infty, x))=\int_{y^{-1}((-\infty, x))} H_{\xi} d \xi=\int_{-\infty}^{g(x)} H_{\xi} d \xi=H(g(x)) .
$$

Since $X \in F_{0}$ and $y+H=\mathrm{Id}$, we have

$$
\mu((-\infty, x))+x=g(x) .
$$

From the definition of $\bar{y}$, it follows that

$$
\bar{y}(\xi)=\sup \{x \in R \mid g(x)<\xi\} .
$$


For any given $\xi \in R$, using the fact that $y$ is increasing and (4.4), it follows that $\bar{y}(\xi) \leq y(\xi)$. If $\bar{y}(\xi)<y(\xi)$, there then exists $x$ such that $\bar{y}(\xi)<x<y(\xi)$ and (4.6) implies that $g(x) \geq \xi$. Conversely, since $y$ is increasing, we have $x=y(g(x))<y(\xi)$, which implies that $g(x)<\xi$. This is a contradiction. Hence, we have that $\bar{y}=y$. Since $y+H=\mathrm{Id}$, it follows directly from the definitions that $\bar{H}=H, \bar{U}=U, \bar{V}=V$ and $\bar{N}=N$. Hence, $L \circ M=\operatorname{Id}_{F / G}$.

Let $(z, \mu) \in D$ be given and $(y, U, V, N, H)$ be the representative of $L(z, \mu)$ in $F_{0}$ given by (4.2a)-(4.2c). Then, let $(\bar{z}, \bar{\mu})=M \circ L(z, \mu)$. Let $g$ be defined as before by (4.3). The same computation that leads to (4.5) now gives

$$
\bar{\mu}((-\infty, x))+x=g(x) .
$$

Given $\xi \in R$, we consider an increasing sequence $x_{i}$ converging to $y(\xi)$, which is guaranteed by (4.2a), and such that $\mu\left(\left(-\infty, x_{i}\right)\right)+x_{i}<\xi$. Let $i$ tend to infinity. Since $F(x)=\mu((-\infty, x))$ is lower semi-continuous, we have $\mu((-\infty, y(\xi)))+y(\xi) \leq \xi$. Take $\xi=g(x)$ and then we get

$$
\mu((-\infty, x))+x \leq g(x)
$$

By the definition of $g$, there exists an increasing sequence $\xi_{i}$ converging to $g(x)$ such that $y\left(\xi_{i}\right)<x$. It follows from the definition of $y$ in (4.2a) that $\mu((-\infty, x))+x \geq \xi_{i}$. Passing to the limit, we obtain $\mu((-\infty, x))+x \geq g(x)$ which, together with (4.8), yields

$$
\mu((-\infty, x))+x=g(x) .
$$

We obtain that $\bar{\mu}=\mu$ by comparing (4.9) and (4.7). It is clear from the definitions that $\bar{z}=z$. Hence, $(\bar{z}, \bar{\mu})=(z, \mu)$ and $M \circ L=\operatorname{Id}_{D}$.

Our next task is to transport the topology defined in $F / G$ into $D$, which is guaranteed by the fact that we have established a bijection between the two equivalent systems and then obtained a continuous semigroup of solutions for the original system.

Let us define the distance $d_{D}$ on $D$ as

$$
d_{D}((z, \mu),(\bar{z}, \bar{\mu}))=d_{F / G}(L(z, \mu), L(\bar{z}, \bar{\mu}))
$$

which makes the bijection $L$ between $D$ and $F / G$ into an isometry. Since $F / G$ equipped with $d_{F / G}$ is a complete metric space, it is not hard to know that $D$ equipped with the metric $d_{D}$ is also a complete metric space. For each $t \in R$, we define the mapping $T_{t}: D \rightarrow D$ as

$$
T_{t}=M \tilde{S}_{t} L
$$

Theorem 4.2 Given $(\bar{z}, \bar{\mu}) \in D$, if we denote $t \rightarrow(z, \mu)(t)=T_{t}(\bar{z}, \bar{\mu})$ the corresponding trajectory, then $z=(u, v)$ is a weak solution of the two-component Camassa-Holm equations (2.1), which constructs a continuous semigroup. Moreover, $\mu$ is a weak solution of the following transport equation:

$$
\mu_{t}+(u \mu)_{x}=\left(u^{3}-2 P u\right)_{x} .
$$

Furthermore, we have

$$
\mu(t)(R)=\mu(0)(R) \quad \text { for all } t
$$


and

$$
\begin{aligned}
\mu(t)(R) & =\mu_{\mathrm{ac}}(t)(R)=\|z(t)\|_{H^{1}}^{2} \\
& =\|u(t)\|_{H^{1}}^{2}+\|v(t)\|_{L^{2}}^{2}=\mu(0)(R) \quad \text { for almost all } t .
\end{aligned}
$$

Thus, the unique solution described here is a conservative weak solution of system (2.1).

Proof To prove that $z=(u, v)$ is a weak solution of the original system (2.1), it suffices to show that, for all $\phi \in C^{\infty}\left(R^{+} \times R\right)$ with compact support,

$$
\begin{aligned}
& \int_{R^{+} \times R}\left(-u \phi_{t}+u u_{x} \phi\right)(t, x) d x d t=-\int_{R^{+} \times R}\left(P_{x} \phi\right)(t, x) d x d t \\
& \int_{R^{+} \times R}\left(-v \phi_{t}+u v_{x} \phi\right)(t, x) d x d t=-\int_{R^{+} \times R}\left[(v+1) u_{x} \phi\right](t, x) d x d t
\end{aligned}
$$

where $P_{x}$ is given by (2.1). We denote by the solution $(y, U, V, N, H)(t)$ of $(2.10)$ a representative of $L(z(t), \mu(t))$. On the one hand, since $y(t, \xi)$ is Lipschitz and invertible w.r.t. $\xi$ for almost all $t$, we can use the change of variables $x=y(t, \xi)$, then we get

$$
\begin{aligned}
& \int_{R^{+} \times R}\left(-u \phi_{t}+u u_{x} \phi\right)(t, x) d x d t \\
& \quad=\int_{R^{+} \times R}\left[-\left(U y_{\xi}\right)(t, \xi) \phi_{t}(t, y(t, \xi))+\left(U U_{\xi}\right)(t, \xi) \phi(t, y(t, \xi))\right] d \xi d t
\end{aligned}
$$

Since $y_{t}=U$ and $y_{\xi t}=U_{\xi}$, it then follows from (2.10) that

$$
\begin{aligned}
\int_{R^{+} \times R} & {\left[-U y_{\xi} \phi_{t}(t, y)+U U_{\xi} \phi(t, y)\right] d \xi d t } \\
= & \frac{1}{4} \int_{R^{+} \times R^{2}}\left\{\operatorname{sgn}\left(\xi-\xi^{\prime}\right) e^{-\left|y(\xi)-y\left(\xi^{\prime}\right)\right|}\left[H_{\xi}+\left(U^{2}+2 V\right) y_{\xi}\right]\right\}\left(\xi^{\prime}\right) \\
& \cdot \phi(t, y(\xi)) y_{\xi}(\xi) d \xi^{\prime} d \xi d t .
\end{aligned}
$$

On the other hand, using the change of variables $x=y(t, \xi)$ and $x^{\prime}=y\left(t, \xi^{\prime}\right)$, and since $y$ is an increasing function, we have

$$
\begin{aligned}
& -\int_{R^{+} \times R}\left(P_{x} \phi\right)(t, x) d x d t \\
& =\frac{1}{2} \int_{R^{+} \times R^{2}}\left[\operatorname{sgn}\left(\xi-\xi^{\prime}\right) e^{-\left|y(\xi)-y\left(\xi^{\prime}\right)\right|}\left(u^{2}+\frac{1}{2} u_{x}^{2}+\frac{1}{2} v^{2}+v\right)\right]\left(t, y\left(\xi^{\prime}\right)\right) \\
& \quad \cdot \phi(t, y(\xi)) y_{\xi}\left(\xi^{\prime}\right) y_{\xi}(\xi) d \xi^{\prime} d \xi d t .
\end{aligned}
$$

It follows from the identity (3.5c) that

$$
\begin{aligned}
& -\int_{R^{+} \times R}\left(P_{x} \phi\right)(t, x) d x d t \\
& =\frac{1}{4} \int_{R^{+} \times R^{2}}\left\{\operatorname{sgn}\left(\xi-\xi^{\prime}\right) e^{-\left|y(\xi)-y\left(\xi^{\prime}\right)\right|}\left[H_{\xi}+\left(U^{2}+2 V\right) y_{\xi}\right]\right\}\left(\xi^{\prime}\right) \\
& \quad \cdot \phi(t, y(\xi)) y_{\xi}(\xi) d \xi^{\prime} d \xi d t .
\end{aligned}
$$


By comparing (4.15) and (4.16), we know that

$$
\int_{R^{+} \times R}\left[-U y_{\xi} \phi_{t}(t, y)+U U_{\xi} \phi(t, y)\right] d \xi d t=-\int_{R^{+} \times R}\left(P_{x} \phi\right)(t, x) d x d t
$$

Thus, the first identity in (4.13) follows directly from (4.14) and the second identity in (4.13) follows in the same way. It is not hard to check that $\mu(t)$ is the solution of (4.10). From the definition $\mu$ in (4.1b), we can get that

$$
\mu(t)(R)=\int_{R} H_{\xi} d \xi=H(t, \infty)
$$

which is constant in time from Lemma 3.3(iii). Thus, we have proved (4.11).

Since $y_{\xi}(t, \xi)>0$ a.e. for almost every $\xi \in R$, it then follows from (3.5c) that

$$
\mu(t)(B)=\int_{y^{-1}(B)} H_{\xi} d \xi=\int_{y^{-1}(B)}\left(U^{2}+U_{\xi}^{2} / y_{\xi}^{2}+V^{2}\right) y_{\xi} d \xi
$$

for any Borel set $B$. Since $y$ is one-to-one and $u_{x} \circ y y_{\xi}=U_{\xi}$ almost everywhere, then (4.17) implies that

$$
\mu(t)(B)=\int_{B}\left(u^{2}+u_{x}^{2}+v^{2}\right)(t, x) d x
$$

Thus, (4.12) holds and the proof is completed.

\section{Multipeakon solutions of the original system}

We derive a new system of ordinary differential equations for the multipeakon solutions which is well posed even when collisions occur in this section, and the variables $(y, U, V, N, H)$ are used to characterize multipeakons in a way that avoids the problems related to blowing up.

Solutions of the two-component Camassa-Holm system may experience wave breaking in the sense that the solution develops singularities in finite time, while keeping the $H^{1}$ norm finite. Extending the solution beyond wave breaking imposes significant challenge as can be illustrated in the case of multipeakons given by

$$
u(t, x)=\sum_{i=1}^{n} p_{i}(t) e^{-\left|x-q_{i}(t)\right|}
$$

where $\left(p_{i}(t), q_{i}(t)\right)$ satisfy the explicit system of ordinary differential equations

$$
\left\{\begin{array}{l}
\dot{p}_{i}=\sum_{j=1}^{n} p_{i} p_{j} \operatorname{sgn}\left(q_{i}-q_{j}\right) e^{-\left|q_{i}-q_{j}\right|} \\
\dot{q}_{i}=\sum_{j=1}^{n} p_{j} e^{-\left|q_{i}-q_{j}\right|}
\end{array}\right.
$$

Peakons interact in a way similar to that of solitons of the $\mathrm{CH}$ equation, and wave breaking may appear when at least two of the $q_{i}$ coincide. Clearly, if the $q_{i}$ remain distinct, system (5.2) allows for a global smooth solution. In the case where $p_{i}(0)$ has the same sign for all $i=1,2, \ldots, n$, the $q_{i}(t)$ remain distinct, and (5.2) admits a unique global solution. In this 
case, the peakons are traveling in the same direction. However, when two peakons have opposite signs, collisions may occur, and if so, system (5.2) blows up.

We consider initial data $\bar{z}=(\bar{u}, \bar{v})$ given by

$$
\left\{\begin{array}{l}
\bar{u}(x)=\sum_{i=1}^{n} p_{i} e^{-\left|x-\xi_{i}\right|}, \\
\bar{v}(x)=\sum_{i=1}^{n} r_{i} e^{-\left|x-\xi_{i}\right|} .
\end{array}\right.
$$

Without loss of generality, we assume that the $p_{i}$ and $r_{i}$ are all nonzero, and that the $\xi_{i}$ are all distinct. From Theorem 4.2 we know that there exists a unique and global weak solution with initial data (5.3), and the aim is to characterize this solution explicitly. We consider the following characterization of multipeakons. The multipeakons are given as continuous solutions $u$ defined on intervals $\left[x_{i}, x_{i+1}\right]$ as the solutions of the Dirichlet problem

$$
u-u_{x x}=0, \quad u\left(x_{i}\right)=u_{i}, \quad u\left(x_{i+1}\right)=u_{i+1},
$$

where the variables $x_{i}$ denote the position of the peaks, and the variables $u_{i}$ denote the values of $u$ at the peaks. In the following, we will show that this property persists for conservative solutions.

Let us define $\bar{X}=(\bar{y}, \bar{U}, \bar{V}, \bar{N}, \bar{H})$ as

$$
\begin{aligned}
& \bar{y}(\xi)=\xi, \\
& \bar{U}(\xi)=\bar{u}(\xi), \\
& \bar{V}(\xi)=\bar{v}(\xi), \\
& \bar{N}(\xi)=\bar{u}_{x}(\xi), \\
& \bar{H}(\xi)=\int_{-\infty}^{\xi}\left(u^{2}+u_{x}^{2}+v^{2}\right) d x,
\end{aligned}
$$

which is a representative of $z=(u, v)$ in the Lagrangian equivalent system, that is, $[\bar{X}]=$ $L\left(\bar{z},\left(\bar{u}^{2}+\bar{u}_{x}^{2}+\bar{v}^{2}\right) d x\right)$. Let $A=R \backslash\left\{\xi_{1}, \ldots, \xi_{n}\right\}$. We claim that the functions $\bar{U}, \bar{V}, \bar{N}$ and $\bar{H}$ belong to $C^{2}(A)$ and even belong to $C^{\infty}(A)$, as the next lemma shows.

Lemma 5.1 For given initial data $\bar{X}=(\bar{y}, \bar{U}, \bar{V}, \bar{N}, \bar{H}) \in F$ such that $\bar{X} \in\left[C^{2}(A)\right]^{5}$, the associated solution $X=(y, U, V, W, H)$ of $(2.10)$ belongs to $C^{1}\left(R^{+},\left[C^{2}(A)\right]^{5}\right)$.

Proof To prove this lemma, one proceeds as in Theorem 3.1 by using the contraction argument. The Banach space $E$ is replaced by

$$
\bar{E}=E \cap\left[C^{2}(A)\right]^{5}
$$

endowed with the norm

$$
\|X\|_{\bar{E}}=\|X\|_{E}+\|y-\operatorname{Id}\|_{W^{2, \infty}(A)}+\|U\|_{W^{2, \infty}(A)}+\|V\|_{W^{2, \infty}(A)}+\|N\|_{W^{2, \infty}(A)}+\|H\|_{W^{2, \infty}(A)} .
$$

It suffices to show that $P$ and $P_{i}$ are Lipschitz from bounded sets of $\bar{E}$ into $H^{1}(R) \cap C^{2}(A)$. Given a bounded set $B=\left\{X \in \bar{E} \mid\|X\|_{\bar{E}} \leq C_{B}\right\}$, where $C_{B}$ is a positive constant, it follows 
from Lemma 3.2 that

$$
\left\|P_{x}(X)-P_{x}(\bar{X})\right\|_{L^{\infty}(R)} \leq C\|X-\bar{X}\|_{E} \leq C\|X-\bar{X}\|_{\bar{E}}
$$

for a constant $C$ depending only on $C_{B}$. From the derivative of $P_{x}$ given by (3.1) and Lemma 3.1, we have that $P_{x}$ is locally Lipschitz from $\bar{E}$ into $C^{1}(A)$. Similarly, we obtain the same result for $P$. We compute the derivative of $P_{\xi}$ and $P_{x \xi}$ on $A$ as follows:

$$
\begin{aligned}
& P_{x \xi \xi}=-H_{\xi \xi} / 2+\left(P_{x} y_{\xi}-U U_{\xi}-V_{\xi}\right) y_{\xi}+\left(P-U^{2} / 2-V\right) y_{\xi \xi}, \\
& P_{\xi \xi}=-H_{\xi} y_{\xi} / 2+\left(P-U^{2} / 2-V\right) y_{\xi}^{2}+P_{x} y_{\xi \xi} .
\end{aligned}
$$

Since $P_{\xi \xi}$ and $P_{x \xi \xi}$ are locally Lipschitz maps from $\bar{E}$ into $C(A)$, we have that $P$ and $P_{x}$ are locally Lipschitz from $\bar{E}$ into $C^{2}(A)$. The local solution of (2.10) in $\bar{E}$ then can be obtained by the standard contraction argument. As we know, as far as global existence is concerned, $\|X\|_{W^{1, \infty}(R)}$ does not blow up with initial data in $W^{1, \infty}(R)$ (see Lemma 3.3(i)). For any $\xi \in A$, we have that

$$
\begin{aligned}
y_{\xi \xi t}= & U_{\xi \xi}, \\
U_{\xi \xi t}= & \frac{1}{2} H_{\xi \xi}-\left(P_{x} y_{\xi}-U U_{\xi}-V_{\xi}\right) y_{\xi}-\left(P-\frac{1}{2} U^{2}-V\right) y_{\xi \xi}, \\
V_{\xi \xi t}= & -V_{\xi \xi} N-2 V_{\xi} N_{\xi}-V N_{\xi \xi}-N_{\xi \xi}, \\
N_{\xi \xi t}= & 2 U_{\xi}^{2}+2 U U_{\xi}-N_{\xi}^{2}-N N_{\xi \xi}+V_{\xi}^{2}+V V_{\xi \xi}+V_{\xi \xi}+\frac{1}{2} H_{\xi} y_{\xi} \\
& -\left(P-\frac{1}{2} U^{2}-V\right) y_{\xi}^{2}+P_{x} y_{\xi \xi}, \\
H_{\xi \xi t}= & \left(3 U^{2}-2 P\right) U_{\xi \xi}-2 U P_{x} y_{\xi \xi}+6 U U_{\xi}^{2}-4 U_{\xi} P_{x} y_{\xi} \\
& +U H_{\xi} y_{\xi}-2 U P y_{\xi}^{2}+U^{3} y_{\xi}^{2}+2 U V y_{\xi}^{2} .
\end{aligned}
$$

System (5.6) is affine w.r.t. $y_{\xi \xi}, U_{\xi \xi}, V_{\xi \xi}, N_{\xi \xi}, H_{\xi \xi}$. Hence, on any interval $[0, T)$, we have

$$
\left\|X_{\xi \xi}(t, \cdot)\right\|_{L^{\infty}(A)} \leq\left\|X_{\xi \xi}(0, \cdot)\right\|_{L^{\infty}(A)}+C+C \int_{0}^{t}\left\|X_{\xi \xi}(\tau, \cdot)\right\|_{L^{\infty}(A)} d \tau,
$$

where $C$ is a constant depending only on $\sup _{t \in[0, T)}\|X\|_{W^{1, \infty}(R)}$, which is bounded. Thus $\|X\|_{W^{2, \infty}(A)}$ does not blow up from Gronwall's lemma, and therefore the solution is globally defined in $\bar{E}$.

Theorem 5.1 Let the initial data be given in (5.3). The solution given by Theorem 4.2 satisfies $u-u_{x x}=0$ between the peaks.

Proof Assuming that $y_{\xi}(t, \xi) \neq 0$, we have

$$
u_{x} \circ y=\frac{U_{\xi}}{y_{\xi}}, \quad u_{x x} \circ y=\left(\frac{U_{\xi}}{y_{\xi}}\right)_{\xi} \frac{1}{y_{\xi}}=\frac{U_{\xi \xi} y_{\xi}-y_{\xi \xi} U_{\xi}}{y_{\xi}^{3}} .
$$


Hence,

$$
\left(u-u_{x x}\right) \circ y=\left(U y_{\xi}^{3}-U_{\xi \xi} y_{\xi}+y_{\xi \xi} U_{\xi}\right) / y_{\xi}^{3} .
$$

Let

$$
M=U y_{\xi}^{3}-U_{\xi \xi} y_{\xi}+y_{\xi \xi} U_{\xi}
$$

For a given $\xi \in A$, differentiating (5.8) w.r.t. $t$, we obtain, by using (2.10), (2.11) and (5.6), that

$$
\begin{aligned}
d M / d t= & 3 U y_{\xi}^{2} y_{\xi t}+U_{t} y_{\xi}^{3}-U_{\xi \xi t} y_{\xi}-U_{\xi \xi} y_{\xi t}+y_{\xi \xi t} U_{\xi}+y_{\xi \xi} U_{\xi t} \\
= & -P_{x} y_{\xi}^{3}+3 y_{\xi}^{2} U U_{\xi}-H_{\xi \xi} y_{\xi} / 2+\left(Q y_{\xi}-U U_{\xi}+A U_{\xi}-V_{\xi}\right) y_{\xi}^{2} \\
& +\left(P-U^{2} / 2-V\right) y_{\xi \xi} y_{\xi}-U_{\xi \xi} U_{\xi}+U_{\xi \xi} U_{\xi}+y_{\xi \xi}\left[H_{\xi} / 2+\left(U^{2} / 2+V-P\right) y_{\xi}\right] \\
= & 2 U U_{\xi} y_{\xi}^{2}-H_{\xi \xi} y_{\xi} / 2+H_{\xi} y_{\xi \xi} / 2-V_{\xi} y_{\xi}^{2} .
\end{aligned}
$$

We differentiate (3.5c) w.r.t. $\xi$ and get

$$
y_{\xi \xi} H_{\xi}+y_{\xi} H_{\xi \xi}=2 y_{\xi} y_{\xi \xi} U^{2}+2 y_{\xi}^{2} U U_{\xi}+2 U_{\xi} U_{\xi \xi}+2 y_{\xi} y_{\xi \xi} V^{2}+2 y_{\xi}^{2} V V_{\xi}
$$

After inserting the value of $y_{\xi} H_{\xi \xi}$ given by (5.10) into (5.9) and multiplying the equation by $y_{\xi}$, we obtain that

$$
y_{\xi} \cdot d M / d t=U U_{\xi} y_{\xi}^{3}+U_{\xi}^{2} y_{\xi \xi}-U_{\xi} U_{\xi \xi} y_{\xi}
$$

It follows from (3.5c) and since $y_{\xi t}=U_{\xi}$ that

$$
y_{\xi} \cdot d M / d t=y_{\xi t} \cdot M
$$

We claim that $M / y_{\xi}$ is $C^{1}$ in time. Indeed, we have

$$
\frac{M}{y_{\xi}}=U y_{\xi}^{2}-U_{\xi \xi}+\frac{y_{\xi \xi} U_{\xi}}{y_{\xi}}=U y_{\xi}^{2}-U_{\xi \xi}+\frac{y_{\xi \xi} U_{\xi}}{y_{\xi}+H_{\xi}}+\frac{y_{\xi \xi} N H_{\xi}}{y_{\xi}+H_{\xi}}=\frac{J\left(X, X_{\xi}, X_{\xi \xi}\right)}{y_{\xi}+H_{\xi}}
$$

for some polynomial $J$. Since $X \in C^{1}(R, \bar{E})$, we have $X, X_{\xi}$ and $X_{\xi \xi}$ are $C^{1}$ in time. Since $X(t)$ remains in $\Gamma$ for all $t$, from (3.5b), we have $y_{\xi}+H_{\xi}>0$ and therefore $1 /\left(y_{\xi}+H_{\xi}\right)$ is $C^{1}$ in time, which implies that $M / y_{\xi}$ is $C^{1}$ in time. For any time $t$ such that $y_{\xi}(t) \neq 0$, we have

$$
\frac{d}{d t}\left(\frac{M}{y_{\xi}}\right)=\frac{M_{t} y_{\xi}-y_{\xi t} M}{y_{\xi}^{2}}=0
$$

Hence,

$$
M(t, \xi)=K(\xi) y_{\xi}(t, \xi)
$$


for some constant $K(\xi)$ independent of time. This leads to

$$
y_{\xi}^{2}\left(u-u_{x x}\right) \circ y=K(\xi)
$$

which corresponds to the conservation of spatial angular momentum. For the multipeakons at time $t=0$, we have $y(0, \xi)=\xi$ and $\left(u-u_{x x}\right)(0, \xi)=0$ for all $\xi \in A$. Hence,

$$
M / y_{\xi}(t, \xi)=0
$$

for all time $t$ and all $\xi \in A$. Thus, $\left(u-u_{x x}\right)(t, \xi)=0$.

For solutions with multipeakon initial data, we have the following result: If $y_{\xi}(t, \xi)$ vanishes at some point $\bar{\xi}$ in the interval $\left(\xi_{i}, \xi_{i+1}\right)$, then $y_{\xi}(t, \xi)$ vanishes everywhere in $\left(\xi_{i}, \xi_{i+1}\right)$. Furthermore, for the given initial multipeakon solution $\bar{z}(x)=(\bar{u}, \bar{v})(x)=$ $\left(\sum_{i=1}^{n} p_{i} e^{-\left|x-\xi_{i}\right|}, \sum_{i=1}^{n} r_{i} e^{-\left|x-\xi_{i}\right|}\right)$, let $(y, U, V, N, H)$ be the solution of system (2.10) with initial data $(\bar{y}, \bar{U}, \bar{V}, \bar{N}, \bar{H})$ given by (5.4a)-(5.4e), then between adjacent peaks, if $x_{i}=y\left(t, \xi_{i}\right) \neq$ $x_{i+1}=y\left(t, \xi_{i+1}\right)$, the solution $z(t, x)=(u, v)(t, x)$ is twice differentiable with respect to the space variable, and for $x \in\left(x_{i}, x_{i+1}\right)$, we have that $\left(u-u_{x x}\right)=0$.

We now start the derivation of a system of ordinary differential equations for multipeakons.

For each $i=1,2, \ldots, n$, we have, from (2.10), that

$$
\left\{\begin{array}{l}
d y_{i} / d t=u_{i}, \\
d u_{i} / d t=-P_{x i}, \\
d v_{i} / d t=-\left(v_{i}+1\right) u_{x i}, \\
d u_{x i} / d t=u_{i}^{2}-u_{x i}^{2} / 2+v_{i}^{2} / 2+v_{i}-P_{i}, \\
d H_{i} / d t=u_{i}^{3}-2 u_{i} P_{i},
\end{array}\right.
$$

where $\left(y_{i}, u_{i}, v_{i}, u_{x i}, H_{i}\right)=(y, U, V, N, H)\left(t, \xi_{i}\right), P_{i}=P\left(t, \xi_{i}\right), P_{x i}=P_{x}\left(t, \xi_{i}\right)$, respectively. By using the change of variables $x=y(t, \xi), P_{i}$ and $P_{x i}$ can be rewritten as

$$
\begin{aligned}
& P_{i}=1 / 2 \cdot \int_{R} e^{-\left|y_{i}-x\right|}\left(u^{2}+u_{x}^{2} / 2+v^{2} / 2+v\right) d x, \\
& P_{x i}=-1 / 2 \cdot \int_{R} \operatorname{sgn}\left(y_{i}-x\right) e^{-\left|y_{i}-x\right|}\left(u^{2}+u_{x}^{2} / 2+v^{2} / 2+v\right) d x .
\end{aligned}
$$

For $x \in\left[y_{i}, y_{i+1}\right], i=1,2, \ldots, n-1$, we write $z=(u, v)$ as

$$
z(x)=\left(\begin{array}{l}
u(x) \\
v(x)
\end{array}\right)=\left(\begin{array}{l}
A_{i} e^{x}+B_{i} e^{-x} \\
C_{i} e^{x}+D_{i} e^{-x}
\end{array}\right) .
$$

The constants $A_{i}, B_{i}, C_{i}$ and $D_{i}$ depend on $u_{i}, u_{i+1}, v_{i}, v_{i+1}, y_{i}$ and $y_{i+1}$ and read

$$
\begin{aligned}
A_{i}=\frac{e^{-\bar{y}_{i}}}{2}\left[\frac{\bar{u}_{i}}{\cosh \left(\delta y_{i}\right)}+\frac{\delta u_{i}}{\sinh \left(\delta y_{i}\right)}\right], & B_{i}=\frac{e^{\bar{y}_{i}}}{2}\left[\frac{\bar{u}_{i}}{\cosh \left(\delta y_{i}\right)}-\frac{\delta u_{i}}{\sinh \left(\delta y_{i}\right)}\right], \\
C_{i}=\frac{e^{-\bar{y}_{i}}}{2}\left[\frac{\bar{v}_{i}}{\cosh \left(\delta y_{i}\right)}+\frac{\delta v_{i}}{\sinh \left(\delta y_{i}\right)}\right], & D_{i}=\frac{e^{\bar{y}_{i}}}{2}\left[\frac{\bar{v}_{i}}{\cosh \left(\delta y_{i}\right)}-\frac{\delta v_{i}}{\sinh \left(\delta y_{i}\right)}\right],
\end{aligned}
$$


where

$$
\begin{array}{ll}
\bar{y}_{i}=\frac{1}{2}\left(y_{i}+y_{i+1}\right), & \delta y_{i}=\frac{1}{2}\left(y_{i}-y_{i+1}\right), \\
\bar{u}_{i}=\frac{1}{2}\left(u_{i}+u_{i+1}\right), & \delta u_{i}=\frac{1}{2}\left(u_{i}-u_{i+1}\right), \\
\bar{v}_{i}=\frac{1}{2}\left(v_{i}+v_{i+1}\right), & \delta v_{i}=\frac{1}{2}\left(v_{i}-v_{i+1}\right) .
\end{array}
$$

The constants $A_{i}, B_{i}, C_{i}$ and $D_{i}$ uniquely determine $z=(u, v)$ on the interval $\left[y_{i}, y_{i+1}\right]$. Thus, we can compute

$$
\begin{aligned}
\delta H_{i}= & H_{i+1}-H_{i}=\int_{y_{i}}^{y_{i+1}}\left(u^{2}+u_{x}^{2}+v^{2}\right) d x \\
= & 2 \bar{u}_{i}^{2} \tanh \left(\delta y_{i}\right)+2 \delta u_{i}^{2} \operatorname{coth}\left(\delta y_{i}\right)+\bar{v}_{i}^{2} \tanh \left(\delta y_{i}\right) \\
& +\delta v_{i}^{2} \operatorname{coth}\left(\delta y_{i}\right)+4 C_{i} D_{i} \delta y_{i} .
\end{aligned}
$$

At this point, we can get some more understanding of what is happening at the time of collision. Let $t^{*}$ be the time when the two peaks located at $y_{i}$ and $y_{i+1}$ collide, i.e., such that $\lim _{t \rightarrow t^{*}} \delta y_{i}(t)=0$. The function $z=(u, v)$ remains continuous because the solution $z=(u, v)$ remains in $H^{1} \times\left[L^{2} \cap L^{\infty}\right]$ for all time, thus we have $\lim _{t \rightarrow t^{*}} \delta u_{i}(t)=\lim _{t \rightarrow t^{*}} \delta v_{i}(t)=0$. Still, $A_{i}, B_{i}, C_{i}$ and $D_{i}$ may have a finite limit when $t$ tends to $t^{*}$. However, the first derivative blows up, which implies $\lim _{t \rightarrow t^{*}} B_{i}=-\lim _{t \rightarrow t^{*}} A_{i}=\infty$ and $\lim _{t \rightarrow t^{*}} D_{i}=-\lim _{t \rightarrow t^{*}} C_{i}=\infty$. Thus $\delta u_{i}$ and $\delta v_{i}$ tend to zero but slower than $\delta y_{i}$. In fact, if we let $t$ tend to $t^{*}$ in (5.18), to first order in $\delta y_{i}$, we obtain

$$
\sqrt{2 \delta u_{i}^{2}+\delta v_{i}^{2}}=\sqrt{\delta H_{i}} \cdot \sqrt{\delta y_{i}}+o\left(\delta y_{i}\right)
$$

which implies that $\delta u_{i}$ and $\delta v_{i}$ tend to zero at the same rate as $\sqrt{\delta y_{i}}$. We now turn to the computation of $P_{i}$ given by (5.14). Let us write $z=(u, v)$ as

$$
\begin{aligned}
z(t, x) & =(u(t, x), v(t, x)) \\
& =\left(\sum_{j=0}^{n}\left(A_{j} e^{x}+B_{j} e^{-x}\right) \chi_{\left(y_{j}, y_{j+1}\right)}(x), \sum_{j=0}^{n}\left(C_{j} e^{x}+D_{j} e^{-x}\right) \chi_{\left(y_{j}, y_{j+1}\right)}(x)\right) .
\end{aligned}
$$

We have set $y_{0}=-\infty, y_{n+1}=\infty, u_{0}=u_{n+1}=0, v_{0}=v_{n+1}=0, A_{0}=u_{1} e^{-y_{1}}, B_{0}=0, A_{n}=0$, $B_{n}=u_{n} e^{y_{n}}$ and $C_{0}=v_{1} e^{-y_{1}}, D_{0}=0, C_{n}=0, D_{n}=v_{n} e^{y_{n}}$. We have

$$
\begin{aligned}
u^{2}+\frac{1}{2} u_{x}^{2}+\frac{1}{2} v^{2}+v= & \sum_{j=0}^{n}\left(\frac{3}{2} A_{j}^{2} e^{2 x}+A_{j} B_{j}+\frac{3}{2} B_{j}^{2} e^{-2 x}\right. \\
& \left.+\frac{1}{2} C_{j}^{2} e^{2 x}+C_{j} D_{j}+\frac{1}{2} D_{j}^{2} e^{-2 x}+C_{j} e^{x}+D_{j} e^{-x}\right) \chi\left(y_{j}, y_{j+1}\right) .
\end{aligned}
$$

Let

$$
k_{i j}= \begin{cases}1 & \text { if } i>j, \\ -1 & \text { if } i \leq j .\end{cases}
$$


Inserting (5.19) into (5.14), we obtain

$$
\begin{aligned}
P_{i}= & \frac{1}{2} \sum_{j=0}^{n} \int_{y_{j}}^{y_{j+1}} e^{-k_{i j}\left(y_{i}-x\right)}\left(\frac{3}{2} A_{j}^{2} e^{2 x}+A_{j} B_{j}+\frac{3}{2} B_{j}^{2} e^{-2 x}\right. \\
& \left.+\frac{1}{2} C_{j}^{2} e^{2 x}+C_{j} D_{j}+\frac{1}{2} D_{j}^{2} e^{-2 x}+C_{j} e^{x}+D_{j} e^{-x}\right) d x .
\end{aligned}
$$

From (5.16) and (5.18), we get

$$
\begin{aligned}
& A_{j}^{2}=\frac{e^{-2 \bar{y}_{j}}}{\sinh ^{2}\left(2 \delta y_{j}\right)}\left[\bar{u}_{j}^{2} \sinh ^{2}\left(\delta y_{j}\right)+2 \bar{u}_{j} \delta u_{j} \sinh \left(\delta y_{j}\right) \cosh \left(\delta y_{j}\right)+\delta u_{j}^{2} \cosh ^{2}\left(\delta y_{j}\right)\right] \\
&=\frac{e^{-2 \bar{y}_{j}}}{2 \sinh \left(2 \delta y_{j}\right)}\left[\bar{u}_{j}^{2} \tanh \left(\delta y_{j}\right)+2 \bar{u}_{j} \delta u_{j}+\delta u_{j}^{2} \operatorname{coth}\left(\delta y_{j}\right)\right] \\
& B_{j}^{2}=\frac{e^{2 \bar{y}_{j}}}{2 \sinh \left(2 \delta y_{j}\right)}\left[\bar{u}_{j}^{2} \tanh \left(\delta y_{j}\right)-2 \bar{u}_{j} \delta u_{j}+\delta u_{j}^{2} \operatorname{coth}\left(\delta y_{j}\right)\right] \\
& A_{j} B_{j}=\frac{1}{4 \sinh \left(2 \delta y_{j}\right)}\left[2 \bar{u}_{j}^{2} \tanh \left(\delta y_{j}\right)-2 \delta u_{j}^{2} \operatorname{coth}\left(\delta y_{j}\right)\right] \\
& C_{j}^{2}= \frac{e^{-2 \bar{y}_{j}}}{2 \sinh \left(2 \delta y_{j}\right)}\left[\bar{v}_{j}^{2} \tanh \left(\delta y_{j}\right)+2 \bar{v}_{j} \delta v_{j}+\delta v_{j}^{2} \operatorname{coth}\left(\delta y_{j}\right)\right] \\
& D_{j}^{2}= \frac{e^{2 \bar{y}_{j}}}{2 \sinh \left(2 \delta y_{j}\right)}\left[\bar{v}_{j}^{2} \tanh \left(\delta y_{j}\right)-2 \bar{v}_{j} \delta v_{j}+\delta v_{j}^{2} \operatorname{coth}\left(\delta y_{j}\right)\right] \\
& C_{j} D_{j}=\frac{1}{4 \sinh \left(2 \delta y_{j}\right)}\left[2 \bar{v}_{j}^{2} \tanh \left(\delta y_{j}\right)-2 \delta v_{j}^{2} \operatorname{coth}\left(\delta y_{j}\right)\right] .
\end{aligned}
$$

It then follows from (5.21)-(5.26) that

$$
\begin{aligned}
\int_{y_{j}}^{y_{j+1}} e^{-k_{i j}\left(y_{i}-x\right)} A_{j}^{2} e^{2 x} d x= & \frac{e^{-k_{i j} y_{i}} \cdot e^{k_{i j} \bar{y}_{j}}}{\left(2+k_{i j}\right) \sinh \left(2 \delta y_{j}\right)} \sinh \left(\left(2+k_{i j}\right) \delta y_{j}\right) \\
& \cdot\left[\bar{u}_{j}^{2} \tanh \left(\delta y_{j}\right)+2 \bar{u}_{j} \delta u_{j}+\delta u_{j}^{2} \operatorname{coth}\left(\delta y_{j}\right)\right], \\
\int_{y_{j}}^{y_{j+1}} e^{-k_{i j}\left(y_{i}-x\right)} B_{j}^{2} e^{-2 x} d x= & \frac{e^{-k_{i j} y_{i}} \cdot e^{k_{i j} \bar{y}_{j}}}{\left(k_{i j}-2\right) \sinh \left(2 \delta y_{j}\right)} \sinh \left(\left(k_{i j}-2\right) \delta y_{j}\right) \\
& \cdot\left[\bar{u}_{j}^{2} \tanh \left(\delta y_{j}\right)-2 \bar{u}_{j} \delta u_{j}+\delta u_{j}^{2} \operatorname{coth}\left(\delta y_{j}\right)\right], \\
\int_{y_{j}}^{y_{j+1}} e^{-k_{i j}\left(y_{i}-x\right)} C_{j}^{2} e^{2 x} d x= & \frac{e^{-k_{i j} y_{i}} \cdot e^{k_{i j} \bar{y}_{j}}}{\left(2+k_{i j}\right) \sinh \left(2 \delta y_{j}\right)} \sinh \left(\left(2+k_{i j}\right) \delta y_{j}\right) \\
& \cdot\left[\bar{v}_{j}^{2} \tanh \left(\delta y_{j}\right)+2 \bar{v}_{j} \delta v_{j}+\delta v_{j}^{2} \operatorname{coth}\left(\delta y_{j}\right)\right], \\
\int_{y_{j}}^{y_{j+1}} e^{-k_{i j}\left(y_{i}-x\right)} D_{j}^{2} e^{-2 x} d x= & \frac{e^{-k_{i j} y_{i}} \cdot e^{k_{i j} \bar{y}_{j}}}{\left(k_{i j}-2\right) \sinh \left(2 \delta y_{j}\right)} \sinh \left(\left(k_{i j}-2\right) \delta y_{j}\right) \\
& \cdot\left[\bar{v}_{j}^{2} \tanh \left(\delta y_{j}\right)-2 \bar{v}_{j} \delta v_{j}+\delta v_{j}^{2} \operatorname{coth}\left(\delta y_{j}\right)\right],
\end{aligned}
$$




$$
\begin{aligned}
\int_{y_{j}}^{y_{j+1}} e^{-k_{i j}\left(y_{i}-x\right)} A_{j} B_{j} d x= & \frac{e^{-k_{i j} y_{i}} \cdot e^{k_{i j} \bar{y}_{j}}}{2 \sinh \left(2 \delta y_{j}\right)} \sinh \left(\delta y_{j}\right) \\
& \cdot\left[2 \bar{u}_{j}^{2} \tanh \left(\delta y_{j}\right)-2 \delta u_{j}^{2} \operatorname{coth}\left(\delta y_{j}\right)\right], \\
\int_{y_{j}}^{y_{j+1}} e^{-k_{i j}\left(y_{i}-x\right)} C_{j} D_{j} d x= & \frac{e^{-k_{i j} y_{i}} \cdot e^{k_{i j} \bar{y}_{j}}}{2 \sinh \left(2 \delta y_{j}\right)} \sinh \left(\delta y_{j}\right) \\
& \cdot\left[2 \bar{v}_{j}^{2} \tanh \left(\delta y_{j}\right)-2 \delta v_{j}^{2} \operatorname{coth}\left(\delta y_{j}\right)\right] .
\end{aligned}
$$

Therefore, the above formulas (5.27)-(5.32) imply that

$$
\begin{aligned}
P_{i}= & \sum_{j=0}^{n} \frac{e^{-k_{i j} y_{i}} \cdot e^{k_{i j} \bar{y}_{j}}}{4 \cosh \left(\delta y_{j}\right)}\left\{\left[\bar{u}_{j}^{2} \tanh \left(\delta y_{j}\right)+\delta u_{j}^{2} \operatorname{coth}\left(\delta y_{j}\right)\right] \cdot 2 \cosh ^{2}\left(\delta y_{j}\right)\right. \\
& +4 k_{i j} \bar{u}_{j} \delta u_{j} \sinh ^{2}\left(\delta y_{j}\right)+2 \bar{u}_{j}^{2} \tanh \left(\delta y_{j}\right)+\frac{2}{3} \sinh ^{2}\left(\delta y_{j}\right) \cdot\left[\bar{v}_{j}^{2} \tanh \left(\delta y_{j}\right)+\delta v_{j}^{2} \operatorname{coth}\left(\delta y_{j}\right)\right] \\
& +\frac{4}{3} k_{i j} \bar{v}_{j} \delta v_{j} \sinh ^{2}\left(\delta y_{j}\right)+2 \bar{v}_{j}^{2} \tanh \left(\delta y_{j}\right) \\
& \left.+2\left[\bar{v}_{j}-k_{i j} \delta v_{j} \operatorname{coth}\left(\delta y_{j}\right)\right] \cdot \delta y_{j}+\left[\bar{v}_{j} \sinh \left(\delta y_{j}\right)+k_{i j} \delta v_{j} \cosh \left(\delta y_{j}\right)\right] \cdot 2 \cosh \left(\delta y_{j}\right)\right\},
\end{aligned}
$$

which can also be written in the following form

$$
P_{i}=\sum_{j=0}^{n} P_{i j}
$$

with

$$
P_{i j}=\left\{\begin{array}{l}
\frac{1}{4} u_{1}^{2} e^{y_{1}-y_{i}}+\frac{1}{12} v_{1}^{2} e^{y_{1}-y_{i}}+\frac{1}{4} v_{1} e^{y_{1}-y_{i}}, \quad \text { for } j=0, \\
\frac{e^{-k_{i j} y_{i} \cdot e_{i j} \bar{i}_{j} \bar{y}_{j}} 4 \cosh \left(\delta y_{j}\right)}{}\left\{\left[\bar{u}_{j}^{2} \tanh \left(\delta y_{j}\right)+\delta u_{j}^{2} \operatorname{coth}\left(\delta y_{j}\right)\right] \cdot 2 \cosh ^{2}\left(\delta y_{j}\right)+4 k_{i j} \bar{u}_{j} \delta u_{j} \sinh ^{2}\left(\delta y_{j}\right)\right. \\
\quad+2 \bar{u}_{j}^{2} \tanh \left(\delta y_{j}\right)+\frac{2}{3} \sinh ^{2}\left(\delta y_{j}\right) \cdot\left[\bar{v}_{j}^{2} \tanh \left(\delta y_{j}\right)+\delta v_{j}^{2} \operatorname{coth}\left(\delta y_{j}\right)\right] \\
\quad+\frac{4}{3} k_{i j} \bar{v}_{j} \delta v_{j} \cdot \sinh ^{2}\left(\delta y_{j}\right)+2 \bar{v}_{j}^{2} \tanh \left(\delta y_{j}\right)+2\left[\bar{v}_{j}-k_{i j} \delta v_{j} \operatorname{coth}\left(\delta y_{j}\right)\right] \cdot \delta y_{j} \\
\left.\quad+\left[\bar{v}_{j} \sinh \left(\delta y_{j}\right)+k_{i j} \delta v_{j} \cosh \left(\delta y_{j}\right)\right] \cdot 2 \cosh \left(\delta y_{j}\right)\right\}, \quad \text { for } j=1, \ldots, n-1, \\
\frac{1}{4} u_{n}^{2} e^{y_{i}-y_{n}}+\frac{1}{12} v_{n}^{2} e^{y_{i}-y_{n}}+\frac{1}{4} v_{n} e^{y_{i}-y_{n}}, \quad \text { for } j=n .
\end{array}\right.
$$

We compute $P_{i x}$ in the same way and obtain

$$
P_{i x}=-\sum_{j=0}^{n} k_{i j} P_{i j}
$$

Now we can summarize the result as follows.

Theorem 5.2 Let $\bar{y}_{i}=\xi_{i},\left(\bar{u}_{i}, \bar{v}_{i}, \bar{u}_{x i}\right)=\left(\bar{u}, \bar{v}, \bar{u}_{x}\right)\left(\xi_{i}\right)$ and $\bar{H}_{i}=\int_{-\infty}^{\xi_{i}}\left(\bar{u}^{2}+\bar{u}_{x}^{2}+\bar{v}^{2}\right) d x$ for $i=$ $1, \ldots, n$ with a multipeakon initial data $\bar{z}=(\bar{u}, \bar{v})$ given by (5.3). Then with initial data $\left(\bar{y}_{i}, \bar{u}_{i}, \bar{v}_{i}, \bar{u}_{x i}, \bar{H}_{i}\right)$ there exists a global solution $\left(y_{i}, u_{i}, v_{i}, u_{x i}, H_{i}\right)$ of (5.13), (5.33), (5.34). For each time $t, u(t, x)$ is defined as the solution of the Dirichlet problem $u-u_{x x}=0$ with boundary conditions $u\left(t, y_{i}(t)\right)=u_{i}(t), u\left(t, y_{i+1}(t)\right)=u_{i+1}(t)$ on each interval $\left[y_{i}(t), y_{i+1}(t)\right]$. Thus, 


\section{$z=(u, v)$ is a conservative solution of the two-component Camassa-Holm system, which is the multipeakon conservative solution.}

\section{Competing interests}

The authors declare that they have no competing interests.

\section{Authors' contributions}

YS conceived of the multipeakon conservative solution of the two-component Camassa-Holm system and participated in its coordination and helped to draft the manuscript. YW participated in the design and coordination of the study, carried out the global conservative solution and the multipeakon conservative solution for the two-component Camassa-Holm system and drafted the manuscript. All authors read and approved the final manuscript.

\section{Acknowledgements}

The paper is supported by the Major State Basic Research Development Program 973 (No. 2012CB215202), the National Natural Science Foundation of China (No. 61134001) and the Fundamental Research Funds for the Central Universities (No. CDJXS12170003). The authors would like to thank the referees for constructive suggestions and comments.

Received: 6 March 2013 Accepted: 26 June 2013 Published: 10 July 2013

\section{References}

1. Fuchssteiner, B, Fokas, AS: Symplectic structures, their Bäcklund transformations and hereditary symmetries. Physica D 4, 47-66 (1981/1982)

2. Camassa, R, Holm, D: An integrable shallow water equation with peaked solitons. Phys. Rev. Lett. 71, 1661-1664 (1993)

3. Constantin, A: On the scattering problem for the Camassa-Holm equation. Proc. R. Soc. Lond., Ser. A, Math. Phys. Eng. Sci. 457, 953-970 (2001)

4. Bressan, A, Constantin, A: Global conservative solutions of the Camassa-Holm equation. Arch. Ration. Mech. Anal. $183,215-239(2007)$

5. Holden, H, Raynaud, X: Global conservative solutions of the Camassa-Holm equation - a Lagrangian point of view. Commun. Partial Differ. Equ. 32, 1511-1549 (2007)

6. Holden, H, Raynaud, X: Global conservative multipeakon solutions of the Camassa-Holm equation. J. Hyperbolic Differ. Equ. 4, 39-64 (2007)

7. Bressan, A, Constantin, A: Global dissipative solutions of the Camassa-Holm equation. Appl. Anal. 5, 1-27 (2007)

8. Pan, C, Jia, L, Cai, R, Ding, Y: Modeling and simulation of channel for underwater communication network. Int. J. Innov. Comput. Inf. Control 8, 2149-2156 (2012)

9. Song, X, Zou, Z, Ye, J: Modeling, design and experiment of improved super-mini underwater robot. Int. J. Innov. Comput. Inf. Control 7, 1109-1120 (2011)

10. Constantin, A: The Hamiltonian structure of the Camassa-Holm equation. Expo. Math. 15, 53-85 (1997)

11. Constantin, A, Lannes, D: The hydrodynamical relevance of the Camassa-Holm and Degasperis-Procesi equations. Arch. Ration. Mech. Anal. 192, 165-186 (2009)

12. Constantin, A, Escher, J: Wave breaking for nonlinear nonlocal shallow water equations. Acta Math. 181, 229-243 (1998)

13. Constantin, A: Global existence of solutions and breaking waves for a shallow water equation: a geometric approach. Ann. Inst. Fourier (Grenoble) 50, 321-362 (2000)

14. Chen, M, Liu, SQ, Zhang, Y: A 2-component generalization of the Camassa-Holm equation and its solutions. Lett. Math. Phys. 75, 1-15 (2006)

15. Falqui, G: On a Camassa-Holm type equation with two dependent variables. J. Phys. A 39, 327-342 (2006)

16. Constantin, A, Ivanov, R: On an integrable two-component Camassa-Holm shallow water system. Phys. Lett. A 372 , 7129-7132 (2008)

17. Johnson, RS: Camassa-Holm, Korteweg de Vries and related models for water waves. J. Fluid Mech. 455, 63-82 (2002)

18. Gui, G, Liu, Y: On the Cauchy problem for the two-component Camassa-Holm system. Math. Z. 268, 45-66 (2011)

19. Escher, J, Lechtenfeld, O, Yin, ZY: Well-posedness and blow-up phenomena for the 2-component Camassa-Holm equation. Discrete Contin. Dyn. Syst. 19, 493-513 (2007)

20. Wang, Y, Huang, J: Global conservative solutions of the two-component Camassa-Holm shallow water system. Int. J. Nonlinear Sci. 9, 379-384 (2010)

21. Guan, C, Yin, ZY: Global existence and blow-up phenomena for an integrable two-component Camassa-Holm shallow water system. J. Differ. Equ. 248, 2003-2014 (2010)

22. Gui, G, Liu, Y: On the global existence and wave-breaking criteria for the two-component Camassa-Holm system. J. Funct. Anal. 258, 4251-4278 (2010)

23. Guan, C, Yin, Z: Global weak solutions for a two-component Camassa-Holm shallow water system. J. Funct. Anal. 260, 1132-1154 (2011)

24. Tian, L, Wang, Y: Global conservative and dissipative solutions of a coupled Camassa-Holm equations. J. Math. Phys. 52, $063702(2011)$

25. Evans, LC, Gariepy, RF: Measure Theory and Fine Properties of Functions. CRC Press, Boca Raton (1992) 